\title{
El portafolio como herramienta que fortalece la reflexión de la práctica en la formación inicial docente
}

\author{
The portfolio as a tool \\ that strengthens reflection on practice \\ in initial teacher training
}

\section{JORGE VALDIVIA GUZMÁN (*a)}

Facultad de Educación de la Universidad de Concepción, Concepción, Bío Bío, Chile

*jvaldivi@udec.cl, https://orcid.org/0000-0001-9011-5415

\section{RESUMEN}

En la formación inicial docente, la adquisición de aprendizajes disciplinares y pedagógicos del futuro profesional se consideran como relevantes al momento de evaluar su proceso formativo en la educación superior, más aún cuando su actuación en la práctica progresiva y final se ve reflejada al momento de responder a las exigencias y necesidades atingentes a la realidad educativa. Lo expuesto se puede evidenciar a través de un portafolio educativo.

El portafolio educativo se define como una colección de recursos impresos o digitales (informes, pautas de trabajo, fotografías, videos, etc.) relacionados, en este caso, con el quehacer pedagógico, y representa una oportunidad para el futuro docente de hacer una reflexión crítica de su accionar con respecto al proceso de enseñanza-aprendizaje, el cual está supeditado a su participación y deliberación al momento de ingresar al centro educativo.

Este artículo busca evidenciar la relevancia del uso del portafolio en la formación inicial docente, en donde la colaboración dialógica entre el profesor y los futuros docentes, y estos últimos 
con los estudiantes, permitan entre otros aspectos promover la práctica reflexiva, el compartir sus logros o experiencias obtenidas de su entorno profesional de modo que el análisis individual y colectivo propenda a mejorar su desempeño en la futura práctica profesional, espacio en donde la autonomía y decisiones pedagógicas son elementos críticos de éxito para su inserción en la educación.

La recopilación de la información documental e investigativa enriquecerá el carácter compilativo argumentativo de la temática en cuestión, con el objeto de exhibir distintas visiones de las aportaciones del portafolio educativo para la praxis del futuro docente, lo que conllevaría a evaluar su incorporación en la formación del pregrado en la Facultad de Educación de la Universidad de Concepción.

Palabras Claves: Profesor (a), portafolio educativo, formación inicial docente, praxis educativa, realidad escolar

\section{SUMMARY}

In initial teacher training, the acquisition of disciplinary and pedagogical learning of the future teacher are considered relevant when evaluating their training process in higher education, especially when their professional performance in progressive and final practice is reflected at the time of respond to the demands and needs related to educational reality. The foregoing can be evidenced through an educational portfolio.
The educational portfolio can be defined as a collection of printed or digital resources (reports, work guidelines, photographs, videos, etc.) related in this case to the pedagogical task, and represents an opportunity for the future teacher to make a critical reflection of their actions regarding the teaching-learning process, which is subject to their participation and deliberation when entering the educational center.

The chapter seeks to demonstrate the relevance of the use of the portfolio in initial teacher training, where the dialogical collaboration between the teacher and the future teachers, and the latter with the students, allow, among other aspects, to promote reflective practice, share their achievements or experiences obtained from their professional environment so that individual and collective analysis can tend to improve their performance in their future professional practice, a space where autonomy and pedagogical decisions are critical elements of success for their insertion in education.

The compilation of information of a documentary and investigative nature will enrich the argumentative compilation nature of the subject in question, with the aim of exhibiting different views on the contributions of the educational portfolio to the praxis of the future teacher, which would certainly lead to evaluating its incorporation in undergraduate training at the Faculty of Education of the University of Concepción.

Key Words: Teacher, educational portfolio, initial teacher training, educational practice, school reality. 


\section{INTRODUCCIÓN}

El desenvolvimiento de los futuros profesores durante su praxis educativa, y la reflexión que ello conlleva, supone un análisis no solo teórico, sino que también comportamental en cuanto a las diversas acciones didácticas que incorporan e implementan en el aula. Los enfoques de enseñanza y su aplicación en el proceso de enseñanza-aprendizaje por parte del futuro docente pueden quedar evidenciados y plasmados en el portafolio educativo, instancia que refleja su actuar en sus prácticas progresivas y finales representadas en sus decisiones pedagógicas y de gestión atribuibles al conocimiento adquirido durante su formación profesional al respecto.

\section{Formación Inicial Docente}

La Formación Inicial Docente (FID) se puede definir como aquella "que, en distintas universidades, imparten las carreras de pedagogía, destinada a preparar a los alumnos para su posterior ejercicio profesional como profesores" (Ministerio de Educación, 2001).

Por otro lado, Reyes (2006, p. 2) explicita el concepto de FID, en función de los alcances de sus componentes esenciales. Es así, como el mismo autor apoyándose en De Leila (1999), expone que por formación "se entiende un proceso permanente de adquisición, estructuración y reestructuración de comportamientos, conocimientos, habilidades y valores para el desempeño de una determinada función, en este caso la función docente". Así mismo, Torres (1996 en Reyes 2006, p. 2), expresa que por inicial y relacionado con el ámbito de la formación docente, es "una formación que se imparte a los futuros educadores después de haber terminado su Enseñanza Media o Secundaria, y por docente se entiende a los educadores que trabajan en el sistema escolar".

En concordancia a lo expuesto, se plantea que la FID:

Es un proceso formativo, crítico y sensible, por lo que significa socialmente, en cualquier iniciativa orientada a mejorar la calidad y equidad de la educación. Es un proceso complejo y multidimensional, ya que junto con dar respuesta a las nuevas demandas e integrar distintas visiones con relación a su diseño o concepción, implementación, desarrollo y evaluación, se concreta en diversos contextos sociales, culturales y educacionales (Reyes, 2006, p. 2)

En este mismo sentido, la FID correspondería a un espacio en donde al futuro docente se le entregan los conocimientos - que harían sinergia con sus habilidades y actitudes - para cumplir de manera eficaz con las exigencias que les plantea el sistema educativo. La conjunción de las variables mencionadas (conocimientos, habilidades y actitudes) permitirán que el profesional de la educación pueda desarrollar competencias de distinta naturaleza - las cuales se evidenciarán a través de su desempeño - y con ello responder de manera óptima a un entorno complejo y multivariable como es el proceso de enseñanza aprendizaje, el cual requiere de estrategias de enseñanzas de tipo participativas y colaborativas, complementadas con acciones que den respuesta a la diversidad de estilos de aprendizajes existentes en el aula. 
Lo anterior es coincidente con el modelo educativo de la Universidad de Concepción (UdeC, 2014), el cual releva una serie de competencias genéricas que deberían incorporarse en los planes de estudio con mayor o menor énfasis según la pertinencia en la carrera.

En efecto, dentro del contexto de su desempeño profesional, se espera que los egresados de la Universidad de Concepción actúen demostrando un conjunto de competencias genéricas, las cuales se presentan agrupadas a continuación (UdeC, 2014):

a) Responsabilidad social, ambiental y compromiso ciudadano

b) Investigación

c) Formación Permanente

d) Administración y Gestión

e) Habilidades Profesionales

De las competencias anteriores se desprenden ámbitos de desempeño que deben ser logrados por los estudiantes de pedagogía durante la FID y evidenciados en el perfil de egreso del Licenciado en Educación (Extraído de la Asignatura de Trabajo de Titulación, 2020). Por citar:

a) Planificar, ejecutar y evaluar procesos de enseñanzaaprendizaje en su área profesional, integrando con una postura innovadora y creativa estrategias y recursos tecnológicos multimodales, según las necesidades de variados contextos educativos.

b) Reflexionar de forma continua sobre su práctica en el sistema educativo respetando la multiculturalidad de los contextos y las diferencias individuales de sus alumnos replanteando progresivamente su desempeño profesional. c) Asumir integralmente su rol docente, afianzado en una dimensión ética de inclusión, respeto, tolerancia y proyección de los valores humanos universales para atender con responsabilidad social a las demandas regionales y nacionales del sistema educativo.

d) Reflexionar y argumentar críticamente sobre bases teórico/ prácticas respecto de la profesión docente, el quehacer educacional y el funcionamiento del sistema educativo.

e) Diseñar, ejecutar y comunicar con rigor científico investigaciones educativas de proyecciones pedagógicas, producto de trabajos en equipos inter y transdisciplinarios.

En este mismo orden y dirección, las competencias que se mencionan son acciones o estrategias que se van consolidando gradualmente durante la FID, y que se pueden evidenciar cuando el estudiante ingresa a sus prácticas progresivas o profesionales al estilo formación dual, lo que conlleva a un crecimiento personal y profesional enriquecedor en su calidad como futuro docente.

Es evidente entonces que existen espacios formales durante la FID, en donde las conversaciones, los debates o controversias surgidas de las prácticas pedagógicas respectivas pueden hacer emerger mejoras o cautelas al momento de implementar la didáctica en la escuela, reflexión significativa y quizás decidora que transformará el modo de llevar a cabo la docencia en su próximo trabajo cotidiano.

En este mismo sentido, se expresa que:

Uno de los objetivos básicos de la formación inicial del profesorado habría de ser el de aprender a pensar de manera crítica. Adoptar una actitud crítica implica ser 
capaz de cuestionar las propias creencias, opiniones, ideas y ser capaz de justificarlas y argumentarlas de forma científica y racional. Por consiguiente, se estima que una de las capacidades a desarrollar durante la formación inicial es la que se ha denominado expresión de opiniones y emisión de juicios (Viscarro, et al. 2000, p. 670)

En efecto, se puede promover el proceso reflexivo en la FID a través del uso del portafolio del futuro profesor, el cual estaría circunscrito a la actuación de este en la escuela, profundizando, por ejemplo, en los elementos que dificultan o hacen posible los procesos educativos, o el compartir experiencias y mejoras de la práctica educativa.

Adicionalmente, por su relevancia para el sistema educativo y, por ende, para evidenciar la actuación de futuro docente en la FID, los argumentos del porqué el portafolio se incorporó en el ámbito escolar se sostiene a que se debió:

A la necesidad de buscar metodologías de naturaleza predominantemente cualitativa para la evaluación de los aprendizajes, de tal manera que el profesor pudiera valorar el proceso del desarrollo de los aprendizajes de cada estudiante, profundizando en la identificación de sus dificultades particulares, de sus habilidades y destrezas, logrando dar retroalimentación y seguimiento a la adquisición de conocimientos y procedimientos complejos, incorporando además el valor añadido de reflexionar sobre su proceso y aumentar su potencial de aprendizaje (Prendes y Sánchez, 2008, p. 24).

Cano (2005), manifiesta que muchas universidades han promovido el proceso reflexivo de los docentes a través de la implementación del portafolio del profesor. Entre los beneficios más destacados del uso del portafolio docente señala tres:

a) Ofrece una oportunidad para la reflexión sobre los objetivos de la enseñanza, la relación profesor-alumno y la eficacia de las estrategias de enseñanza

b) Promueve el diálogo profesional entre pares sobre la enseñanza

c) Es un medio eficaz para propiciar un proceso de reflexión y aprendizaje sobre su rol docente y su enseñanza

Ante las situaciones educativas planteadas, y en donde las opiniones y la manifestación tácita de la capacidad para tomar decisiones y emitir juicios pueden evidenciarse a través de un portafolio, habría que preguntarse entonces:

a) ¿̇En qué consiste?

b) ¿Cuáles son sus ventajas o desventajas para el sistema educativo?

c) ¿Será un medio que permita propiciar una reflexión sobre el proceso de enseñanza-aprendizaje del cual es parte el docente que se encuentra en su pleno proceso de formación? 


\section{PROBLEMA DE INVESTIGACIÓN}

La incorporación del portafolio educativo durante el desarrollo de la práctica educativa de los estudiantes de las carreras de pedagogía en la Facultad de Educación no es una estrategia recurrente para ir evaluando el desempeño profesional de los futuros docentes en la organización escolar.

Resulta oportuno entonces profundizar sobre las potencialidades del portafolio educativo y experiencias existentes en la literatura en el ámbito de la educación, así como también los factores críticos de éxito para su implementación en el entramado curricular existente en la actualidad, en especial en las postrimerías de las carreras de pedagogía.

La recopilación de la información de naturaleza documental e investigativa enriquecerá el carácter compilativo argumentativo de la temática en cuestión, con el objeto de exhibir distintas visiones de las aportaciones del portafolio educativo para la praxis del futuro docente, lo que conllevaría a evaluar su incorporación en la formación del pregrado en la Facultad de Educación de la Universidad de Concepción.

\section{OBJETIVO}

Exponer la relevancia de la incorporación del portafolio como un espacio dialógico para el futuro docente de modo que con el conocimiento obtenido pueda sustentar las bases para una reflexión crítica sobre su práctica educativa y desarrollo profesional.

\section{MARCO CONCEPTUAL}

\section{¿Qué es un Portafolio?}

Las definiciones de portafolio de acuerdo con la literatura son muy diversas. Algunas hacen alusión a la reflexión de la práctica, como una herramienta de almacenamiento de información, o de evidencias emocionales o de sentimientos afectos al docente.

Con respecto al portafolio desde una mirada docente se expresa que "es una colección de información y datos sobre la práctica del profesor, seleccionada por él mismo, donde se evidencia su concepción educativa, desarrollo profesional, experiencias y fortalezas como profesor, y es útil para una variedad de propósitos (Wolf y Dietz, 1998, p. 9).

Además, Rodríguez y Rodríguez (2014, p. 60), comentan que un portafolio "presenta un conjunto de evidencias (documentos, artefactos) organizadas en una estructura que responda a sus propósitos y a determinados criterios o estándares clave, que ayudan a exponer sus competencias profesionales y su quehacer docente".

Cabe agregar, que "[...] el portafolio proporciona la estructura necesaria para documentar y reflexionar sobre la práctica de la docencia y el aprendizaje" (KlenowsKi, 2005, p. 53).

Asimismo, algunos autores, mencionan que el portafolio es: 
Una colección de documentos que pueden ser mostrados como evidencias del proceso de aprendizaje y de los logros de un sujeto. En ese contexto tiene la doble función de:

a) Recoger y reflejar las experiencias de aprendizaje y logros más significativos de una persona (estudiante, profesional, trabajador...) de forma continuada.

b) Informar de forma clara sobre el nivel de competencia y de otras experiencias importantes a lo largo de su aprendizaje o de su carrera. (Barberà, Gewerc y Rodríguez, 2009, p. 6).

Por otro lado, se define al portafolio como:

Un instrumento que tiene como objetivo común la selección de muestras de trabajo o evidencias de consecución de objetivos personales o profesionales que, ordenados y presentados de un determinado modo, cumplen la función de potenciar la reflexión sobre cada una de las prácticas (educativas, profesionales o civiles) (Barberà, Bautista, Espasa y Guasch, 2006, p. 56).

Según se ha citado, el portafolio educativo es un registro documental (impreso o digital) que evidenciaría los avances o dificultades del futuro docente relacionado con su desempeño profesional o competencia asociada (básica, genérica - específica) en la institución escolar. La información y conocimiento desplegado en esta oportunidad, le permitiría al futuro docente reflexionar sobre su práctica pedagógica con la finalidad de mejorar su actuación en el contexto educativo.
De igual forma, y con relación al proceso de reflexión, Klenowski (2005, citado en Rodrígues, 2013), subraya que la elaboración, el uso y la construcción de un portafolio posibilitan el aprendizaje de muchas habilidades y competencias cognitivas. Entre estas, destaca el desarrollo del pensamiento reflexivo.

Según el mismo autor, "el método de evaluación del portafolio tiene implicaciones pedagógicas que influyen en el desarrollo de la reflexión, las cuales consisten en tener la capacidad de revisar de forma crítica y reflexiva los propios procesos y prácticas de aprendizaje". Implica mirar hacia atrás sobre lo hecho y realizado, como ilustran Tartwijk, Driesen, Vleuten y Stokkin (2007, citado en Rodrígues, 2013).

Es evidente entonces el valor implícito que tiene el portafolio en el sentido de fomentar la reflexión en función de lo planificado e implementado según el objetivo a conseguir, aspecto que se valora de manera fundamental, más aún cuando este permite mejorar de manera gradual el desempeño del sujeto en su contexto personal y/o profesional.

De los planteamientos expresados, cabe citar aquí que el portafolio también sería una instancia en donde se destacaría la figura del profesor guía como orientador del accionar del futuro docente cuando la situación lo amerite, refrendado por las evidencias consignadas en esta herramienta de apoyo a la labor pedagógica. Lo expuesto se debería a la interacción bidireccional: futuro docente-profesor guía / futuro docenteportafolio / profesor guía-portafolio. 
Figura 1

El portafolio y su relación con la reflexión de la práctica.

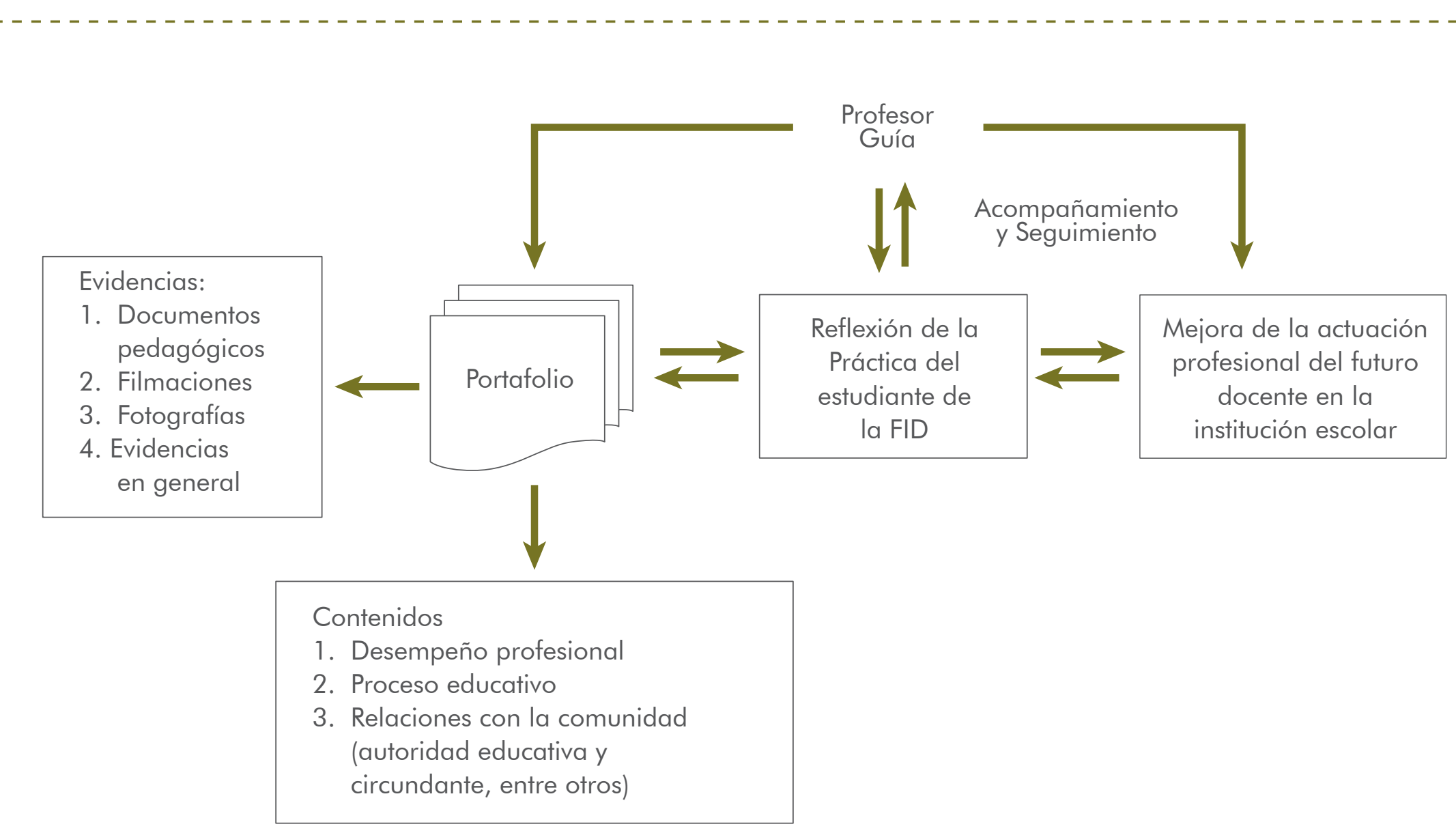

SISTEMA ESCOLAR Y UNIVERSITARIO

\section{SISTEMA ESCOLAR Y UNIVERSITARIO}

Nota. Interacción bidireccional: futuro docente-profesor guía / futuro docente-portafolio / profesor guía-portafolio. Elaborado por el autor. 


\section{Del objetivo del Portafolio}

De acuerdo con Rodrígues (2013), "un portafolio: debe contar con un objetivo claramente definido, pues es este el referente para su elaboración y, por ende, el criterio indispensable para establecer su contenido".

Al mismo tiempo, Zubizarreta (2004) explica que "los portafolios varían en propósito, y los diferentes propósitos determinarán los diversos contenidos".

\section{De la estructura del Portafolio}

En lo que se refiera a este apartado se plantea que:

Un portafolio que cuenta con un objetivo claro, que evidencia el desarrollo de un proceso, que cuenta con una serie de documentos y objetos diversos, debe contar con una estructura, con una organización interna definida por el autor 0 , en algunos casos, por el profesor que orienta el desarrollo del portafolio. Son, por supuesto, estructuras que pueden ser muy distintas, puesto que dependen de muchos factores y de manera especial de la creatividad y de la decisión de su autor sobre cómo quiere organizar y divulgar los documentos coleccionados. Esta sería una sexta característica de los portafolios (Rodrígues, 2013, p. 162).

\section{La reflexión de la práctica como un espacio dialógico en el contexto de la Formación Inicial Docente}

El desarrollo del docente está circunscrito a una diversidad de fenómenos que suceden en el complejo escenario como es el proceso de enseñanza-aprendizaje, que involucran necesariamente a los estudiantes, padres y apoderados, directivos y a la organización escolar propiamente tal, que hacen que en algunas oportunidades surjan conflictos o incidentes críticos de variada índole que requieran de parte del docente la comprensión del evento, y una toma de decisiones de manera oportuna.

Al respecto, Richards y Lockhart (1998) expresan que "el profesor que tenga un conocimiento más extenso y una consciencia más profunda de los distintos componentes y dimensiones de la enseñanza estará mejor preparado para hacer juicios apropiados y tomar decisiones". Al mismo tiempo López (2013) cita que, "en el difícil camino de tomar conciencia y profundizar en esta compleja realidad que es el aula, el papel que puede jugar la reflexión, la autoexploración y la autoevaluación en el desarrollo profesional de los docentes ha abierto nuevas posibilidades acerca de cómo entender la formación inicial del profesorado".

Del mismo modo, Richards y Lockhart (1998) definen el fenómeno reflexivo como "un enfoque en el que profesores expertos y noveles recogen datos acerca de su labor, examinan sus actitudes, creencias, presuposiciones y práctica docente, y utilizan la información obtenida como base para la reflexión crítica sobre la enseñanza". De hecho, como explicita López (2013), "la práctica, y las cuestiones que de ella emergen a través de la reflexión, se perfilan como el punto de partida para iniciar acciones futuras de mejora". 
Cabe agregar que la relevancia del fenómeno reflexivo se pone de manifiesto en:

Esta visión activa del docente en la que es protagonista de su propio desarrollo ya fue enfatizada por Dewey (1933) quien defendía un tipo de profesor intelectualmente responsable y comprometido con su práctica. En las ideas dewinianas subyace un tipo de profesional que es al mismo tiempo fuente y destinatario del conocimiento generado, todo ello a través de la reflexión y con el objetivo de guiar al individuo al establecimiento de nuevas acciones (López, 2013, p. 12).

Ante la situación planteada y de acuerdo con López (1983), Schön (1983), interesado por seguir profundizando en lo que respecta a reflexión profesional, identificó tres conceptos basales para comprender este fenómeno:

a) Conocimiento en la acción. Se trata de un conocimiento que no procede de la actividad intelectual, sino que más bien se origina en la capacidad de adaptación que poseemos los seres humanos al aprender nuevas destrezas. Un ejemplo, sería el uso por parte del docente de la herramienta ZOOM para impartir clases a distancia, lo cual implica una serie de acciones para adaptarse a este contexto, con el desafío de no sólo interactuar con los estudiantes de manera verbal, sino que también concretar acciones tendientes a compartir un documento digital. Lo expuesto tendría por finalidad crear un ambiente semejante a la sesión de clase para desarrollar el proceso formativo, lo que conlleva por defecto al docente a aprender nuevas destrezas en esta oportunidad. b) Reflexión en acción. Es la capacidad de reflexión que se tiene mientras van sucediendo los acontecimientos en el ámbito de la enseñanza, y hace referencia a lo que hacen los docentes cuando se encuentran en el aula: así como van sucediendo los hechos, se actúa y se modifica la planificación de la clase para poder atender a las nuevas necesidades y dificultades surgidas en la misma práctica.

c) Reflexión sobre la acción. La cual requiere del docente para ir un paso más allá de la mera toma de conciencia en la práctica. Se trata de un momento posterior a la sesión de clase, en donde el docente examina lo acontecido en el aula a través de un diario de registro, o en una breve conversación con un colega. El docente, al verbalizar, reflexiona y se convierte en un investigador de su práctica con el objetivo de mejorarla.

Figura 2

Elementos componentes del fenómeno reflexivo

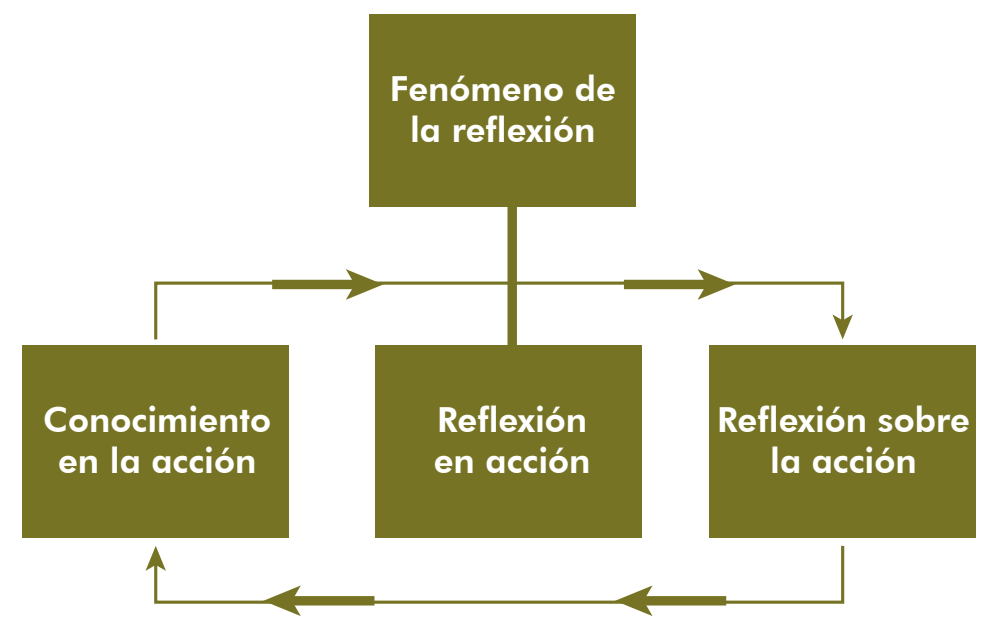

Nota. Elaborado por el autor 
De acuerdo con lo visto, y coincidente con Dewey (1933) y Schön (1983), la reflexión crítica de la enseñanza surge a partir del conocimiento en la acción que involucra procesos de adaptación o de empoderamiento conceptual del docente a los nuevos escenarios educativos, que hace que adquiera habilidades (cognitivas, afectivas y motrices) para dar respuesta a las diversas problemáticas emergentes acaecidas durante su desarrollo profesional. Esto da lugar a un encadenamiento que le da sentido a la reflexión en acción, en donde esta adaptación le permite al docente ir adecuando su programa curricular y de gestión diseñado previamente de modo que haga sinergia con los nuevos eventos educativos, respondiendo con ello no solo a las necesidades vigentes, sino también implementando acciones para la mejora a su práctica educativa. Finalmente, y como producto de la socialización de lo expuesto ante sus pares, aparece la reflexión sobre la acción, que de alguna manera se evidencia a través de notas de campo u otro medio de registro los sucesos acontecidos en el aula, transformándose el mismo docente en un indagador de su práctica educativa con el objeto de mejorar su desempeño profesional. Lo manifestado es un continuo o ciclo a lo largo del accionar del docente en la organización escolar que le permite, por tanto, sistematizar su práctica educativa cada vez más frecuente, optimizando su labor educativa.

Es evidente entonces que:

A lo largo de su vida profesional, los docentes acumulan experiencias (tanto de su etapa de estudiantes como de la de profesor) que se plasman en un conocimiento tácito de su profesión, el llamado conocimiento en la acción. Este conocimiento en la acción se va modificando y reestructurando de manera continua al pensar acerca de su práctica, ya que inevitablemente van sucediendo cosas en el aula que les hacen reflexionar en la acción. Los docentes están teorizando constantemente en sus clases, así como van apareciendo los problemas (Zeichner y Liston, 1987; en Latorre, 1992). Para pasar al siguiente nivel, la reflexión sobre la acción se requiere del profesor una toma de conciencia diferente en la que sean capaces de hablar sobre lo sucedido. Este aspecto de toma de conciencia que parece ser tan relevante en los procesos reflexivos ha sido tratado por varios autores (López, 2013, p. 12).

En López (2013), se comenta que "el concepto de práctica reflexiva tiene diferentes formas de ser definido, y es utilizado en el discurso desde diversos paradigmas educativos".

La misma autora, plantea que "en la caracterización realizada por Ward y MacCotter (2004), citado por Roca y Manchón (2006), ellos exponen tres características esenciales de la actividad reflexiva, a saber:

a) Que la reflexión se contextualiza en la práctica (conjunto de experiencias sobre las que reflexionar);

b) Que la reflexión en la enseñanza tiene un carácter cíclico, (el educador parte de sus teorías personales como base para sustentar su práctica. Estas experiencias posteriormente darán lugar a nuevas reflexiones y probablemente a nuevas teorías)

c) Por último, la reflexión debe buscar múltiples perspectivas; es decir, situarse en el contraste de opiniones y posturas, además de la propia. 
En este mismo orden y dirección, Larrivee (2008) define cuatro niveles de reflexión que son importantes destacar por su enfoque teórico que conlleva a comprender el ámbito de la práctica reflexiva de manera más detallada:

Tabla 1.

Niveles de reflexión de la práctica docente

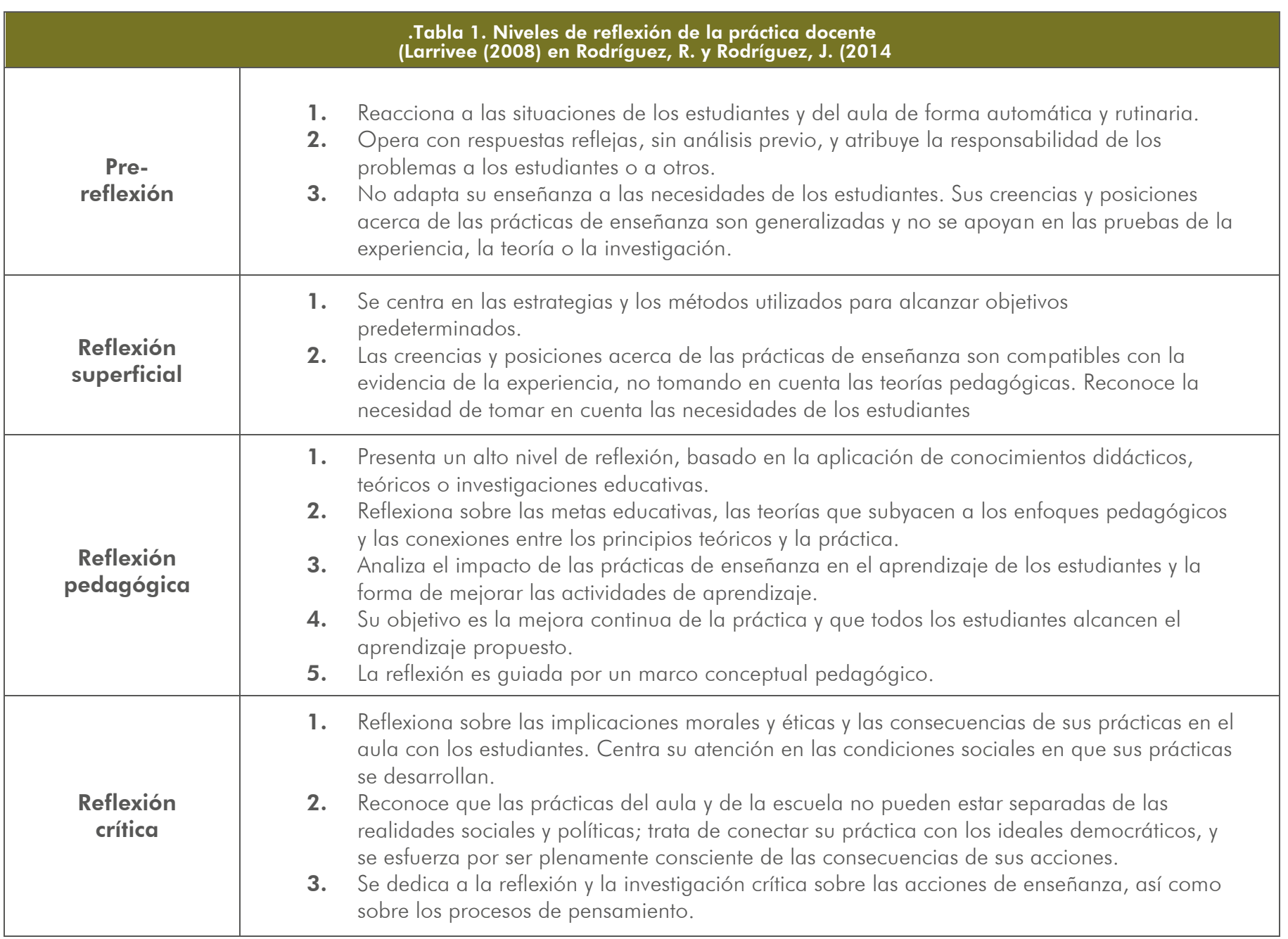


La reflexión de la práctica y su relación con el portafolio docente

Las coincidencias existentes en el fenómeno de la reflexión en la práctica educativa (Dewey, 1933; Schön, 1983; Richards y Lockhart,1998; Larrivee, 2008) con el portafolio educativo, espacio en donde el progreso y desarrollo del profesional queda evidenciado también a través de una reflexión supeditada a la práctica educativa, puede construirse con base en los diferentes enfoques teóricos existentes en la actualidad. No obstante, se considera que la propuesta de Larrivee (2008) es la más afín, en el sentido de disponer de niveles descriptivos que involucran a otras propuestas, y atiende manera decidida aspectos tales como: el análisis crítico de la enseñanza, sus percepciones, sus reflexiones, y los cambios que debe asumir el docente para adaptarse a la organización escolar.

Resulta oportuno mencionar que la propuesta teórica de Schön (1983) también puede considerarse tan válida como la anterior para la implementación de un portafolio, más aún cuando la reflexión en la práctica educativa trae consigo una serie de etapas que son claves para la mejora del desempeño profesional. Es así como la reflexión en y sobre la práctica conllevan la ejecución de un plan de acción, el cual permitirá dar respuesta a las necesidades detectadas, en especial en la reflexión en la práctica a través del enfoque formativo fundado en la investigación-acción que propone Schön (1983).

Sobre la base de las consideraciones anteriores, Barbera (2006) comenta que el portafolio "es un instrumento que tiene como objetivo común la selección de muestras de trabajos o evidencias de consecución de objetivos personales o profesionales que, ordenados y presentados de un determinado modo, cumplen la función de potenciar la reflexión sobre cada una de las prácticas (educativas, profesionales o civiles)". Así, el estudiante en un determinado tiempo en nuestro caso las prácticas profesionales; puede organizar una selección de evidencias (reflexiones, vídeos, entrevistas, fotos, elaboración de instrumentos, etc.) que le permitan, por una parte, demostrar que está aprendiendo, así como, facilitar a su profesor hacer un seguimiento de su progreso.

En efecto:

La reflexión acerca de la práctica educativa también destaca como un factor fundamental que impacta positivamente en la formación docente y en el aprendizaje de los estudiantes (ZeicHner y Liston, 1987; Hatton y SmitH, 1995; Lyons, 1999; Larrivee, 2000; Kane, SanDretto y HeatH, 2004). Muchas universidades han promovido el proceso reflexivo de los docentes a través de la implementación del portafolio del profesor (cano, 2005). Entre los beneficios más destacados del uso del portafolio docente señalan tres: ofrece una oportunidad para la reflexión sobre los objetivos de la enseñanza, la relación profesor-alumno y la eficacia de las estrategias de enseñanza; promueve el diálogo profesional entre pares sobre la enseñanza, y es un medio eficaz para propiciar un proceso de reflexión y aprendizaje sobre su rol docente y su enseñanza (López, 2013, p. 12). 


\section{El diseño de un portafolio educativo}

Varios autores han trabajado en portafolios reflexivos a lo largo de la última década (Barberà, 2006; Esteve, Carandell y Keim 2006, Pujolà y González 2008, entre otros) como herramientas para evidenciar la reflexión sobre la acción (Schön, 1983) de tal forma que el docente pueda profundizar en los fenómenos acontecidos en el aula y actuar de nuevo con un plan de acción definido.

Pujolá y González (2008) elaboraron un Portafolio Reflexivo del Profesor (PRP) en donde se decidieron por una herramienta diseñada para ser usada por los docentes de forma autónoma y a lo largo de toda su formación continua. Cabe destacar que este portafolio considera como base el enfoque formativo sustentado en la investigación-acción que propone Schön (1983).

En López (2013), se explicita que Pujolá y González (2008) definen la estructura de su PRP en seis apartados que en palabras de sus autores "no pretenden encorsetar el proceso, sino más bien ayudar a su elaboración, por lo que pueden adaptarse a las necesidades de cada profesor".

Como vemos en la Figura 3, y en López, (2013), el PRP tiene la siguiente estructura:

a) Sección "Punto de partida", que hace alusión a un "autorretrato" del profesor en el que este se sitúa en el momento actual en cuanto a una serie de temas: formación, experiencia y sus creencias sobre el proceso enseñanza-aprendizaje. i. En la misma sección se encuentra otro apartado denominado "¿̇ónde estoy?" que permite al profesor expresar sus logros, necesidades e inquietudes. En resumen, la primera sección consigue establecer una fotografía del docente en el periodo formativo iniciado que será referente futuro al que remitirse para evaluar su progreso.

b) †Sección "Objetivos", la cual está centrada en la explicitación de objetivos que cada docente decide marcarse para este periodo. Los objetivos deben tener en cuenta el contexto social en el que cada profesor desarrolla su práctica, de tal manera que cada uno de ellos pueda plantearse metas realistas y al mismo tiempo, motivadoras.

c) Sección "Repertorio de Muestras", es la sección más amplia del portafolio, en donde el docente va recogiendo evidencias de su práctica (en forma de documentos, actividades, resultados de los alumnos, fotografías, notas, planes de clase, etc.), acompañándolas con textos reflexivos que intentan indagar en el porqué de la elección de la muestra.

d) Sección "Visión Global", que le permite al autor del portafolio revisar de nuevo los objetivos propuestos al comienzo del uso de la herramienta y reflexionar acerca de su evolución y progreso a lo largo del tiempo.

De acuerdo con lo que se expresa en López (2013), la propuesta de Pujolá y González (2008) "es abierta, de tal modo que los docentes pueden realizar el PRP de forma individual o compartir las experiencias con otros profesionales, bien sea en su propia comunidad de práctica o bien a través de las nuevas posibilidades de comunicación que nos abren las nuevas tecnologías". 


\section{MÉTODO}

Tabla 2.

Estructura del Portafolio Reflexivo del Profesor

\begin{tabular}{|c|c|c|}
\hline \multicolumn{3}{|c|}{$\begin{array}{l}\text { Tabla 2. Estructura del Portafolio Reflexivo del Profesor } \\
\text { (Pujolá y Gonzólez, 2009) }\end{array}$} \\
\hline Introducción & \multicolumn{2}{|c|}{$\begin{array}{l}\text { Mi Portafolio Reflexivo del Profesor. Presentación } \\
\text { del portafolio y sus partes }\end{array}$} \\
\hline $\begin{array}{l}\text { Punto de } \\
\text { Partida }\end{array}$ & $\begin{array}{l}\text { ¿Quién soy? } \\
\text { Formación } \\
\text { Experiencia } \\
\text { Creencias } \\
\text { Rol del profesor } \\
\text { Rol del alumno } \\
\text { Procesos } \\
\text { Aprendizaje / adquisición } \\
\text { Práctica docente }\end{array}$ & $\begin{array}{l}\text { ¿Dónde estoy? } \\
\text { Logros } \\
\text { Necesidades } \\
\text { Inquietudes } \\
\quad \text { Preocupaciones } \\
\text { Curiosidades }\end{array}$ \\
\hline Objetivos & \multicolumn{2}{|l|}{$\begin{array}{c}\text { ¿Adónde voy? } \\
\text { Objetivos } \\
\text { Plan de Acción }\end{array}$} \\
\hline \multirow{2}{*}{$\begin{array}{l}\text { Repertorio } \\
\text { de Muestras }\end{array}$} & Muestras de logros & $\begin{array}{l}\text { Reflexión. ¿̇Cómo he } \\
\text { Ilegado hasta aquí? }\end{array}$ \\
\hline & $\begin{array}{l}\text { Muestras de procesos de } \\
\text { los objetivos marcados }\end{array}$ & $\begin{array}{l}\text { Reflexión. iYa estoy } \\
\text { en camino! }\end{array}$ \\
\hline $\begin{array}{l}\text { Visión } \\
\text { Global }\end{array}$ & \multicolumn{2}{|c|}{$\begin{array}{l}\text { Mi evolución } \\
\text { Evaluación general de mi desarrollo profesional }\end{array}$} \\
\hline
\end{tabular}

Nota. Pujolá y González, 2009
El artículo se adscribe al paradigma interpretativo de carácter fenomenológico que tiene por finalidad comprender la realidad educativa y sus contextos relacionados. En este caso, se desea destacar la incorporación del portafolio con intencionalidad pedagógica en la formación inicial del futuro profesor, el cual participa activamente en su práctica progresiva o profesional, etapas adscritas a los momentos intermedios o finales de su carrera, y en donde la reflexión de la práctica se manifiesta a través de evidencias o compilaciones de los trabajos realizados por ellos durante el período citado, sus inquietudes, sus temores, sus aciertos en lo referente al proceso de aprendizaje individual, supeditados al proceso de enseñanza aprendizaje.

La mirada descriptiva-cualitativa del aporte del portafolio al proceso educativo se sustenta en las aportaciones teóricas de diferentes autores, los cuales dan respuestas a través de este paradigma a las preguntas enunciadas en sus inicios, como son:

a) ¿En qué consiste el portafolio educativo?

b) ¿Cuáles son sus ventajas o desventajas para el sistema educativo?

c) ¿Será un medio que permita propiciar una reflexión sobre el proceso de enseñanza-aprendizaje del cual es parte el docente que se encuentra en su pleno proceso de formación?

Cabe mencionar que la mirada cualitativa tiene por objetivo reconstruir la realidad tal como la observan los actores de un sistema social (Corbetta, 2003). Lo expuesto permite, por 
tanto, evaluar el desarrollo natural de los sucesos, el cual queda evidenciado a través de las aportaciones del futuro profesor en este portafolio, marcando hitos formativos durante su desarrollo profesional.

Lo anteriormente analizado tiene sinergia con la mirada holística que se propone en este estudio al considerar los eventos o sucesos que surgen en las prácticas progresivas o finales como un todo, sin reducir la investigación teórica a sus partes separadas, incorporando con ello no solo el contexto de dichos eventos, sino que la significación de estos a través de la interpretación subjetiva que le da el futuro docente a los hechos que van emergiendo en el transcurso de su práctica educativa.

\section{DISCUSIÓN Y CONCLUSIONES}

Se plantea que uno de los objetivos básicos de la Formación Inicial Docente (FID) es el de fomentar en el futuro profesor el proceso de pensar de manera crítica en lo que respecta a su desarrollo personal, así como también aquello que acontece en su práctica profesional, implicando el cuestionamiento de sus propias creencias, opiniones e ideas con la finalidad de ser capaz de justificarlas y sustentarlas de forma racional antes sus pares (Viscarro, et al. 2000).

Ahora bien, la incorporación del portafolio educativo en la enseñanza universitaria representa un espacio que coincide con lo expuesto, más aún cuando esta colección de documentos y artefactos organizados con un propósito y criterios determinados no solo promueve el proceso reflexivo del rol del futuro docente en el sistema educativo, sino que también se induce el diálogo profesional entre sus pares y el profesor guía sobre el fenómeno de la enseñanza (Cano, 2005).

En efecto, el proceso reflexivo es un elemento importante del andamiaje pedagógico que se debe fortalecer durante la FID, pues los futuros docentes están recogiendo información de su propia práctica, como también examinando sus actitudes y cuestionando sus conocimientos previos, lo que conlleva a ser un punto de partida para implementar acciones de mejora de su propia práctica profesional. En otras palabras, el futuro docente está definiendo y estructurando su propio proyecto personal y profesional a través de la reflexión crítica, y con ello modificar su actuar en lo que respecta al proceso de enseñanza. Es en este marco en donde el portafolio educativo representaría otro componente de este andamiaje pedagógico que permitiría evidenciar las diferentes etapas del crecimiento personal y profesional del futuro docente, así como también las acciones remediales construidas a través del conflicto cognitivo adquirido en su práctica profesional.

Adicionalmente, un tema que debe estar en la discusión y en la definición entre los académicos de la Facultad de Educación, sería el establecer el diseño del portafolio educativo y su posterior implementación en la FID. Para ello existen distintos enfoques teóricos (Pujolá y González, 2008; Barberà, 2005; Lara, Pereira, Alvarado y Muñoz, 2014) que pueden servir de referencia para la construcción colectiva y multidisciplinaria del 
portafolio educativo. Cada uno de ellos tienen sus maneras de recopilar información de lo que sucede en un contexto determinado. Si se lograse definir al portafolio educativo como una herramienta de apoyo y de sistematización para fomentar la reflexión de la práctica del futuro docente, lo más relevante sería que hiciera sinergia no solo con el modelo educativo de la Universidad de Concepción (en este caso), sino que también con el perfil de egreso del Licenciado en Educación, ámbitos de desempeño que pueden quedar evidenciados de manera manifiesta en esta herramienta educativa.

Las aportaciones de los niveles de reflexión de Schön (1983) y de Larrivee (2008) marcan un hallazgo destacado en lo que respecta a la epistemología asociada a la implementación del portafolio en la práctica educativa, pues el fenómeno de la reflexión que se evidencia a través del portafolio educativo abarca todos los estadios de la implementación de la práctica educativa durante la FID (conocimiento en la acción, reflexión en la acción y reflexión sobre la acción), aspectos que deberían considerarse estratégicamente en las prácticas progresivas y profesionales de los futuros docentes.

Finalmente, se puede expresar que la implementación del portafolio en la FID le permitirá al futuro docente reflexionar sobre su práctica educativa, transformándose en un investigador de su propio accionar a través de un plan de mejora de carácter cíclico, dando respuestas a las necesidades detectadas a través del enfoque formativo fundado como es la investigación-acción (Schön, 1983).

\section{REFERENCIAS}

Barberà, E. (2005). La evaluación de competencias complejas. Revista Educere, v31, pp. 497-504.

Barberà, E., Bautista, G., Espasa, A. y Guasch, T. (2006). Portafolio electrónico: desarrollo de competencias profesionales en la Red. Revista de Universidad y Sociedad del Conocimiento (RUSC). 3(2). UOC. Disponible en http://rusc.uoc.edu/rusc/ca/index. $\mathrm{php/rusc/article/download/v3n2-barbera-bautista-espasa-}$ guasch/287-1205-2-PB.pdf

Barberà, E., Gewerc, A. y Rodríguez, J.L. (2009). Portafolios electrónicos y educación superior en España. RED: Revista de Educación a Distancia. Número monográfico VIII. Número especial dedicado a Portafolios electrónicos y educación superior. Disponible en https://www.um.es/ead/red/M8/

Cano, E. (2005). El Portafolios del Profesorado Universitario: Un Instrumento para la Evaluación y para el Desarrollo Profesional (1 a ed.). Barcelona: Octaedro.

Corbetta, P. (2003). Metodología y técnicas de investigación social. McGraw-Hill Interamericana de España (2 ed.). ISBN 978-84481-8399-8

De Leila, C. (1999). Modelos y tendencias de la Formación Docente. Revista Organización de Estados Americanos. Organización Estados Iberoamericanos. Disponible en https://www.oei.es/ historico/cayetano.htm

Dewey, J. (1933). How we think: A restatement of the relation of reflective thinking to the educative process. Chicago: Henry Regnery 
Gomes, M. J. (2008). Educational potential of e-portfolios: from student learning to teacher professional development. En M. J. Loureiro, A. Moreira, \& M. J. Gomes (eds.), ePortfolios and eArgumentation (pp. 13 - 20). Coimbra: Associação de Professores de Sintra. Disponible en http://repositorium.sdum. uminho.pt/handle/1822/8622

Klenowski, V. (2005). Desarrollo de portafolios para el aprendizaje y la evaluación. Madrid: Narcea.

López, S. (2013). Propuesta de portafolio de formación inicial de profesores (PIP): Análisis de las reflexiones de profesores de ELE en formación inicial. Universidad de Barcelona. Disponible en http://diposit.ub.edu/dspace/bitstream/2445/132458/1/tfm_ silvia lopez lopez.pdf

Lara, B.; Pereira, M.; Alvarado, P. y Muñoz, C. (2014). Reflexión de la práctica pedagógica, a través de un portafolio electrónico, en la formación inicial docente. Universidad Católica de El Salvador. Revista Conocimiento Educativo, ISSN 231 1, 2, pp. 21-34.

Larrivee, B. (2000). Transforming teaching practice: becoming the critically reflective teacher. Reflective Practice, 1 (3), pp. 293-307.

Latorre, M. A. (1992). La reflexión en la formación del profesorado. Tesis doctoral. Barcelona: Facultad de Pedagogía de la Universidad de Barcelona.

Murillo, G. (2012). El portafolio como instrumento clave para la evaluación en la educación superior. Revista Electrónica, Actualidades Investigativas en Educación. 12(1), pp. 1-23. Disponible en https://www.redalyc.org/comocitar. oa? id $=44723363015$
Pujolá, T.y González, V. (2008). “El uso del portafolio para la autoevaluación continua del profesor". Monográficos Marco ELE, 7

Prendes-Espinosa, M. P. y Sánchez-Vera, M.M. (2008). Portafolio electrónico: posibilidades para los docentes. Pixel-Bit. Revista de Medios y Educación, 32, pp. 11-25.

Seldin, P. (2004). The Teaching Portfolio: A Practical Guide to Improved Performance and Promotion/Tenure Decisions. Bolton, Mass: Anker Pub. Co.

Roca, J. y Manchón, R. (2006). Análisis de la Reflexión y Preparación para la Actividad Reflexiva en el Prácticum de maestro (inglés). España. Revista de Educación. No 342. Disponible en http://www. educacionyfp.gob.es/dam/jcr:d0709196-3212-4577-9882e3d18384945b/re34218-pdf.pdf

Reyes, L. (2006). Formación inicial docente y práctica pedagógica. Biblioteca Digital DIBRI. Universidad Católica Silva Henríquez. Chile. Obra bajo Licencia Attribution-NonCommercial-NoDerivs 3.0 Unported de Creative Commons. Foro Educacional. ISSN-e 0717-2710, v9, pp. 95-108. Disponible en http://bibliotecadigital.ucsh.cl/greenstone/collect/revista 1_old/archives/ HASHbeec.dir/Formacion\%20inicial\%20docente\%20y\%20 practica\%20pedagogica.pdf

Rodrígues, R. (2013). Los portafolios en el ámbito educativo: usos y beneficios. Trabajo de investigación de la tesis doctoral de la autora: "El desarrollo de la práctica reflexiva sobre el quehacer docente, apoyada en el uso de un portafolio digital, en el marco de un programa de formación para académicos de la Universidad Centroamericana de Nicaragua", defendida el 26 de febrero de 2013 en la Universidad de Barcelona. Disponible 
en https://www.researchgate.net/publication/263927730_Los portafolios_en_el_ambito_educativo_usos_y_beneficios

Rodríguez, R. y Rodríguez, José Luis (2014). El portafolio digital como soporte de la práctica reflexiva en la formación docente. Revista Iberoamericana de Educación. ISSN: 1022-6508, v64, pp. 5374. Disponible en https://rieoei.org/RIE/article/view/393

Richards, J. C. y Lockhart, C. (1998). Estrategias de reflexión sobre la enseñanza de idiomas. Madrid: Cambridge University Press

Schön, D. A. (1983). The reflective Practitioner, How Professionals Think in Action. Nueva York: Basic Books. (Ed. Esp.: El profesional reflexivo. Como piensan los profesionales cuando actúan. Barcelona: Paidós, 1998)

Tartwijk, J.V., Driesen, E., Vleuten, C.V.D. y Stokkin, K. (2007). Factors influencing the successful introduction of portfolios. Quality in Higher Education, 13(1), pp. 69-79

Torres, J. (1996). Globalización e interdisciplinariedad: el curriculum integrado. Morata. Madrid

UdeC (2014). Modelo Educativo. Universidad de Concepción. Dirección de Docencia. Disponible en http://docencia.udec.cl/ wp-content/uploads/2014/05/Modelo_educativo_opti.pdf

Velásquez, R. (2007). Rúbrica. Universidad de San Martín de Porres. Disponible en http://es.scribd.com/doc/2905226/Rubricas-deevaluacion

Viscarro, I.; Delgado, C.; y Camps, C. (2000). Actas del XVIII Congreso nacional de Educación Física. La Formación Inicial y Permanente del profesor de Educación Física. v1. Universidad de Castilla-La Mancha. Coordinador Onofre Contreras Jordán. Colección Estudios, pp. 667-678 Disponible en https://books.google.cl/books?id=MpfWFQPhQaAC\&printse $\mathrm{c}=$ frontcover $\# \mathrm{v}=$ onepage $\& \mathrm{q} \& \mathrm{f}=$ false

Ward, J. R.; McCotter, S. S. (2004). Reflection as a visible outcome for preservice teachers, in Teaching and Teacher Education, 20, pp. 243-257

Wolf, K. y Dietz, M. (1998). Teaching portfolios: purposes and possibilities. Teacher Education Quarterly, Winter, pp. 9-22

Zeichner, K.M. Y Liston, D.P. (1987). Teaching student teachers to reflect. Harvard Educational Review 57 (1), pp. 23-47

Zubizarreta, J. (2004). The Learning Portfolio: Reflective practice for improving student learning. San Francisco: Jossey-Bass.

\section{BIBLIOGRAFÍA}

Cu, D.; Gómez, Y.; Maciel, R. y Ramírez, M. (2019). El portafolio de evidencias, una estrategia de enseñanza. I.C. Investig@ción. $\mathrm{N}^{\circ}$ 16. Recuperado de https://instcamp.edu.mx/wp-content/ uploads/2019/11/Ano2019No16-73-95.pdf

Orbea, G.; Cruz-Iglesias, E, y Rekalde-Rodríguez, I. (2019). ¿Cómo puede el portafolios del alumnado ayudar a mejorar las prácticas docentes? Revista Digital de Investigación en Docencia Universitaria. E-ISSN: 2223-2516. Recuperado de https:// revistas.upc.edu.pe/index.php/docencia/article/view/769 
Morales, D. y Tobón, S. (2017). El Portafolio de evidencias como una modalidad de titulación en las escuelas normales. IE Revista de investigación educativa de la REDIECH. vol. 8, núm. 14. Recuperado de https://www.redalyc.org/ jatsRepo/5216/521653267016/521653267016.pdf

Muñoz, L. y Soto, E. (2019). El portafolio digital ¿̇una herramienta para aprender a ser docentes críticos?: Un estudio de casos. Revista Actualidades Investigativas en Educación, 19(3), 1-32. Doi. 10.15517/aie.v19i3.38632. Recuperado de https://www. scielo.sa.cr/pdf/aie/v19n3/1409-4703-aie-19-03-163.pdf

Ramírez-Casas del Valle, L.; Baleriola, E. Sisti, V. (2019). Pedagogía en la era de la rendición de cuentas: análisis del portafolio docente en Chile. Educação \& Sociedade. vol.40 Campinas. Recuperado en https://www.scielo.br/scielo.php?script=sci_ artext\&pid $=$ S0101-73302019000100322

Trejo, H. (2018). Recursos digitales para la elaboración de e-portafolios educativos. Revista de Filosofía, Letras y Humanidades Departamento de Filosofía / Departamento de Letras. Universidad

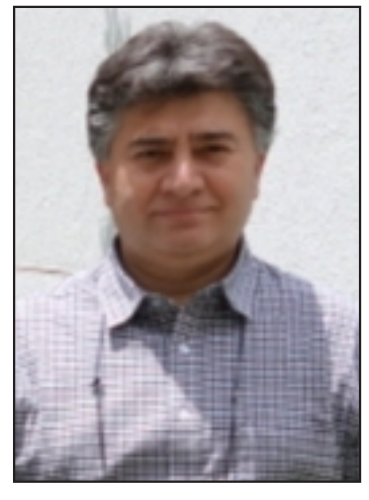

\section{JORGE VALDIVIA GUZMÁN}

- Tiene por objeto evaluar diferentes escenarios TIC para incorporarlas en la educación, como por ejemplo, soluciones e-learning para el profesorado, desarrollo de metodologías participativas usando TIC para la generación de aprendizajes activos y dinámicos, diseños de formación presenciales, entre otros 